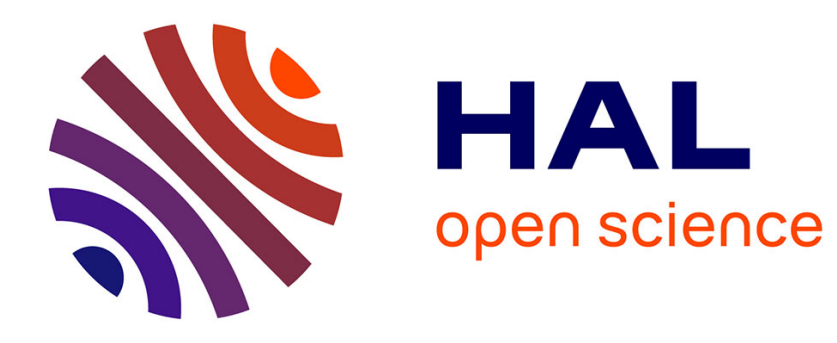

\title{
Une anthropologie historique de la scolastique Sylvain Piron
}

\section{To cite this version:}

Sylvain Piron. Une anthropologie historique de la scolastique. Annales. Histoire, Sciences sociales, 2009, 64 (1), pp.207-215. halshs-00407942

\section{HAL Id: halshs-00407942 https://shs.hal.science/halshs-00407942}

Submitted on 28 Jul 2009

HAL is a multi-disciplinary open access archive for the deposit and dissemination of scientific research documents, whether they are published or not. The documents may come from teaching and research institutions in France or abroad, or from public or private research centers.
L'archive ouverte pluridisciplinaire HAL, est destinée au dépôt et à la diffusion de documents scientifiques de niveau recherche, publiés ou non, émanant des établissements d'enseignement et de recherche français ou étrangers, des laboratoires publics ou privés. 


\section{Une anthropologie historique de la scolastique (note de lecture)}

Paru in Annales Histoire sciences sociales, 64, janvier-février 2009, n 1, p. 207-215.

Sylvain Piron

Cette série de trois ouvrages, réunis sous le titre global de La raison scolastique ${ }^{1}$, marque une inflexion dans la production d'Alain Boureau. Le contraste est net avec une série précédente dont les titres les plus marquants sont Théologie, science et censure au XIII siècle (1999) et Satan hérétique $(2004)^{2}$. Dans ces volumes, l'élaboration de discours théologiques médiévaux sur le corps humain ou la sorcellerie était située dans leurs contextes institutionnels et sociaux, leurs implications politiques et anthropologiques étant longuement étayées. De tels horizons s'estompent ici, sans que le projet d'inscrire la scolastique dans la société médiévale soit pour autant abandonné ; il est seulement laissé en pointillés. Le choix de s'en tenir pour l'essentiel à une lecture interne des œuvres savantes est explicité de façon laconique au début du premier volume. Il tient à une radicalisation de la position illustrée et défendue dans les ouvrages précédents, à savoir qu'une idée « fabrique du réel » (RE, 16). La méthode suivie est elle aussi annoncée sobrement, sans longues justifications. Le choix d'«analyser des textes précis, et peu nombreux, plutôt que de tenter une vue d'ensemble » (RE, 20) a pour avantage revendiqué [208] de mieux faire apparaître l'éclat de quelques formations conceptuelles significatives, là où un panorama exhaustif aurait pu faire la part trop belle à des pensées héritées. Cette option s'accompagne d'un recours prédominant à quelques penseurs, privilégiés en raison de leur inventivité théorique. Au premier rang d'entre eux viennent, dans les trois ouvrages, le dominicain Thomas d'Aquin et le franciscain Pierre de Jean Olivi. Si la présence du premier ne prête guère à discussion, la préférence accordée au second peut surprendre davantage. Outre qu'elle a le mérite de mettre en lumière un auteur important souvent négligé ${ }^{3}$, cette décision me semble produire un gain appréciable. La tension entre ces deux œuvres majeures de la seconde moitié du XIII ${ }^{\mathrm{e}}$ siècle - que l'on peut concevoir comme une véritable « charnière » de la théologie médiévale, aussi bien au sens habituel de l'image que selon la métaphore rugbystique, avec Thomas à la mêlée et Pierre à l'ouverture - construit une polarité qui interdit de réduire l'histoire de la scolastique à un déroulement univoque. De la sorte, le théologien canonisé un demi-siècle après sa mort, dont l'impact culturel fut immense à travers les siècles, n'importe pas davantage qu'un auteur au

\footnotetext{
${ }^{1}$ Alain Boureau, La Religion de l'État. La construction de la République étatique dans le discours théologique de l'Occident médiéval (1250-1350), Paris, Les Belles-Lettres, 2006 ; L'Empire du livre. Pour une histoire du savoir scolastique (1200-1380). La Raison scolastique II, Paris, Les Belles-Lettres, 2007 ; Id., De vagues individus. La condition humaine dans la pensée scolastique. La Raison scolastique III, Paris, Les Belles-Lettres, 2008.

${ }^{2}$ Théologie, science et censure au XIII siècle. Le cas de Jean Peckham, Paris, Les Belles Lettres, 1999 ; Satan hérétique. Naissance de la démonologie dans l'Occident médiéval (1280-1330), Paris, Odile Jacob, 2004.

${ }^{3}$ Cf. Alain Boureau et Sylvain Piron dir., Pierre de Jean Olivi (1248-1298). Pensée scolastique, dissidence spirituelle et société. Actes du colloque de Narbonne (mars 1998), , Paris, Vrin 1999.
} 
destin contrarié, réprouvé peu après son décès, mais qui a ouvert des voies originales et a marqué en profondeur des auteurs cruciaux des générations suivantes, comme Duns Scot ou Ockham.

De ces choix de méthode découle une forme d'écriture kaléidoscopique, qui procède par juxtaposition d'analyses textuelles serrées, dans des rapprochements souvent suggestifs et parfois inattendus. La démarche est entachée d'un double risque, qui n'est qu'en partie assumé : l'accumulation d'études de détail peut faire perdre de vue la perspective générale, d'autant que les moments de synthèse, récapitulant les acquis de la démonstration, se font rares. L'autre difficulté est, quant à elle, pleinement revendiquée. D'autres lectures, une autre sélection de textes ou même un agencement différent des mêmes fragments étudiés auraient pu produire une image d'ensemble méconnaissable. Alain Boureau ne cache pas l'aspect subjectif de son approche de la scolastique (DVI, 16). Pour décrire sa façon de faire, on peut parler d'une lecture créative, engagée dans les débats qu'il éclaire. La richesse foisonnante de ces ouvrages dicte la forme que peut prendre un compte rendu global : plutôt que d'offrir un résumé fidèle des trois volumes et d'entrer dans des discussions de détail, il semble préférable de se contenter de fournir quelques clés de lectures.

Bien que le projet d'une trilogie ait été annoncé dès le premier volume, les trois ouvrages constituent des entités distinctes et peuvent se lire séparément. Le projet a d'ailleurs évolué en cours de route, puisqu'un quatrième et ultime volume est annoncé, qui portera sur l'action morale et judiciaire dans la pensée scolastique. Témoin de ce mouvement interne, on peut aussi noter un glissement du découpage chronologique retenu. En dépit des périodisations annoncées dans les titres, le cœur du travail des deux premiers volumes porte sur les années 1250-1290, prolongé par des incursions, plus ou moins insistantes, dans le XIV siècle. À plusieurs [209] reprises, l'impression qui ressort est qu'une mutation est intervenue autour de 1300 et que le paysage intellectuel ultérieur intéresse moins l'auteur, sans nul jugement dépréciatif sur le fond. Le déplacement qu'opère L'Empire du livre tient à l'intérêt porté à la première génération de théologiens universitaires, actifs dans les années 1220-1230. Le troisième volume, quant à lui, remonte encore plus en amont puisqu'il cherche à suivre le fil d'une question particulière depuis la première moitié du XII ${ }^{\mathrm{e}}$ siècle. Mais à nouveau, c'est la décennie 1220 qui est mise en avant, tandis que le propos ne s'avance pas au-delà de la fin du XIII ${ }^{\mathrm{e}}$ siècle. Alors que la tendance actuelle des historiens de la philosophie médiévale les conduit à se concentrer sur la période postérieure aux grandes condamnations de 1277 , l'un des intérêts de cette trilogie tient au rééquilibrage qu'elle effectue au profit de ces années fondatrices. Quant au cadre disciplinaire, si l'on voit apparaître par moments des maîtres ès arts, en la personne de Siger de Brabant ou de commentateurs anonymes d'Aristote, et plus souvent encore comme un collectif agissant en retrait de l'action principale, la scolastique d'Alain Boureau est principalement une théologie, incorporant l'usage de sources et de techniques philosophiques. Mais si le découpage disciplinaire importe relativement peu, c'est que l'axe de lecture n'est pas structuré 
par les enjeux d'époque, mais par des questionnements propres à l'historien qui, par un détournement revendiqué, cherche à extraire de la théologie savante une réflexion sur l'être humain, son psychisme et son existence collective, une véritable «science de l'homme » comme il est dit plusieurs fois.

Deuxième ouvrage de la série, L'Empire du Livre peut être lu en premier. Il constitue une forme d'introduction à la scolastique qui cherche à illustrer par l'exemple les différentes facettes de l'activité savante. Les aspects institutionnels n'étant que sommairement évoqués, la question prise comme point de départ est celle de la fondation d'une discipline. En dépit de quelques références à theologia comme désignation d'une activité nouvelle à la fin $\mathrm{du} \mathrm{xI}^{\mathrm{e}}$ siècle ou chez Pierre Abélard, l'élément distinctif est l'organisation d'un travail collectif autour d'un texte de référence : les Sentences de Pierre Lombard, recueil d'autorités choisi comme répertoire de questions à débattre au sein de la faculté de théologie. Alain Boureau détourne ici à bon escient la notion de « communauté textuelle », employée par Brian Stock pour caractériser des dissidences fondées autour de l'interprétation d'un texte ${ }^{4}$. Elle permet de désigner une communauté d'interprétation, caractérisée par la confrontation intellectuelle autour d'un référent stable qui permet l'approfondissement et le déplacement graduel des questionnements. Les deux chapitres, qui insistent tour à tour sur le « rassemblement initial » puis sur la « dispersion » produite par l'individualisation de la pensée à l'œuvre dans les commentaires, ne doivent pas se lire comme des moments successifs, mais plutôt comme une tension permanente qui est au centre de la dynamique intellectuelle de la scolastique. Le même Hugues de Saint-Cher sert à illustrer successivement ces deux aspects: alors que sa discussion de la théorie du vœu est étroitement calquée [210] sur celle de Guillaume d'Auvergne, son traitement du sacrement du mariage introduit une innovation lourde de conséquences, en faisant intervenir les catégories aristotéliciennes de matière et forme dans la définition des sacrements. Le maître dominicain peut également incarner la précocité de la «fêlure » au sein de la communauté primitive des maîtres séculiers que constitua l'arrivée des ordres mendiants au sein de l'Université.

Le chapitre consacré à la «raison biblique» présente différentes stratégies d'extraction du sens de l'Écriture, de Pierre le Mangeur à Olivi, en se rattachant à de plus anciens intérêts de l'auteur pour la place du récit dans le christianisme ${ }^{5}$. À sa lumière, on comprend mieux l'usage qui est fait d'analyses exégétiques d'autres lieux de la trilogie, de même que le motif principal d'un relatif désintérêt pour le $\mathrm{XIV}^{\mathrm{e}}$ siècle, qui est justement marqué le découplage de la pratique du commentaire biblique et de la théologie spéculative. Sous le titre de «domination des héritages » sont ensuite associés deux legs de statut bien différent : les autorités patristiques, face auxquelles la

\footnotetext{
${ }^{4}$ Brian Stock, The Implications of Literacy. written language and models of interpretation in the eleventh and twelfth centuries, Princeton (N.J.), Princeton University Press, 1983.

${ }^{5}$ Alain Boureau, L'Evénement sans fin. Récit et christianisme au Moyen Age, Paris, Les Belles Lettres, 1993.
} 
mise à distance est abondamment pratiquée, et la foi dont l'Église est la garante. Les derniers chapitres décrivent quelques techniques intellectuelles : 1'abstraction est illustrée par la difficulté à penser les animaux qui ne sont souvent considérés que dans leur généralité, par différence avec l'humain; en contrepoids, le recours à la pensée par cas témoigne d'une capacité à saisir le singulier. Sous le titre «l'analyse universelle», sont notamment abordées l'attitude face aux traductions et les lectures critiques des maîtres entre eux. Les pages de ce chapitre qui méritent d'être particulièrement mises en valeur concernent les « distinctions du troisième type » (EL, 242257). À partir des années 1280, voire même un peu plus tôt, de nombreux théologiens ont été conduits à élaborer, chacun pour soi, une nouvelle forme de distinction, intermédiaire entre la distinction réelle, qui pointe des différences ontologiques dans les choses mêmes, et la distinction de raison, pure opération de pensée qui ne dit rien du réel. Alain Boureau englobe sous le nom de « distinction d'aspect » des variantes d'une troisième modalité, dont la distinction formelle de Duns Scot est le modèle le plus fameux, qui distingue selon les angles différents sous lesquels les choses se laissent appréhender par la pensée, sans postuler d'altérité réelle. Il y a là une belle saisie, à partir d'un élément technique, d'un basculement majeur de l'histoire intellectuelle médiévale. Ce passage à un rythme ternaire de la pensée permet de saisir un mouvement qui peut être décrit, selon les points de vue, comme déclin de la scolastique ou au contraire comme son renouvellement. Le régime de pensée change en tout cas drastiquement : «Avec la confiance dans le binarisme logique, c'était l'optimisme démonstratif de la scolastique qui s'effondrait » (EL, 257). Olivi n'est pas pour rien au centre du tableau. Sa distinction selon les « raisons réelles », qu'il décrit lui-même comme une distinction selon les «modes d'intelligibilité partielle» des mêmes objets, est l'une des premières solutions de ce type ; les multiples usages qui en sont faits se retrouvent en différents passages des trois [211] volumes de la Raison scolastique. Le dernier chapitre présente un nouveau et dernier balancement entre deux tendances contraires: si des discours collectifs semblent s'imposer, à travers le poids des censures ou la formation d'écoles de pensée, la plasticité de la langue scolastique rend néanmoins possible l'expression de pensées singulières. L'ouvrage se donnait pour but de dissiper les brumes qui entourent la scolastique afin de la faire apparaître comme une intelligence collective, ouverte et créative, mais parcourue de multiples tensions ; le but est atteint.

La Religion de l'État est le plus ambitieux, mais aussi le plus déconcertant des trois ouvrages, en raison du choix de méthode et de la thèse paradoxale qu'il défend. La démarche va à rebours des façons classiques d'écrire l'histoire de la pensée politique. L'essentiel de la réflexion des scolastiques sur le politique ne serait pas à chercher dans des écrits caractérisés par un genre littéraire : «je crois fermement que le genre 'politique' de l'époque incite majoritairement à des platitudes atemporelles ou à des prudences » $(\mathrm{RE}, 17)$; il serait plutôt à dégager de considérations 
théologiques portant sur la divinité et la création, la nature humaine et son destin. Mais autant la suggestion de méthode paraît stimulante, autant les conclusions qui en sont tirées ici semblent excessives. En tirant le fil de ces implications politiques de la théologie scolastique, Alain Boureau voit l'expression d'un concept d' «État-république», élaboré dans la seconde moitié du XIII ${ }^{\mathrm{e}}$ siècle, qui aurait ensuite été contesté autour de 1300, en raison de la prépondérance acquise alors par la notion de souveraineté. La difficulté du propos tient à la nature de l'objet ainsi dégagé. Quelques formules introductives doivent mettre en garde le lecteur. Il ne s'agit pas de faire l'histoire d'un concept explicite mais «l'histoire de l'idée ou du rêve de l'État» (RE, 16), de décrire des « fragments épars, éléments d'une mosaïque pensée mais non réalisée » (RE, 17). Autrement dit, c'est à la latence d'un concept d'État dans la grande scolastique, non exprimé mais à ses yeux nécessairement impliqué par certaines constructions doctrinales, que s'intéresse Alain Boureau. On comprend dès lors ce qui le contraint à forger une expression peu satisfaisante qui ne correspond à aucune formulation d'époque. La «république étatique» mentionnée dans le titre désigne la « forme future et possible de l'État-nation», qui s'en distingue par l'absence de spécification nationale, mais qui réalise déjà pleinement sa définition : «Une réalité politique limitée dans l'espace prend en charge l'universel, censé s'incarner particulièrement dans cette entité close » (RE, 14). La grande difficulté du livre est que cette forme politique abstraite est présentée comme un concept abouti et agissant, alors qu'elle n'est qu'une implication possible et non exprimée d'élaborations théologiques. Il suffit d'opérer cette rectification pour obtenir une présentation de la thèse générale qui me semble mieux correspondre à l'intention de l'auteur. La grande scolastique était porteuse d'un concept possible d'État; autre chose a vu le jour, au moment où le mouvement intellectuel s'est trouvé confronté à des dynamiques politiques effectives. En opérant cette modification, l'essentiel du propos me semble pouvoir être maintenu et même gagner une force d'explication supérieure.

A. Boureau ne situe pas sa démarche par rapport à la théologie politique telle que la conçoit Carl Schmitt (qui n'est pas même mentionné dans le livre), mais il n'est pas inutile de souligner que son propos en prend le contre-pied exact. Loin de [212] fonctionner comme un transfert de concepts théologiques en politique, la politique implicite des scolastiques se définit au contraire par différence et distinction des domaines. Il faut aller à la page 119 pour trouver une expression du point central de la démonstration. Par un effet de structure, la théologie scolastique réclame « un cantonnement religieusement motivé des valeurs proprement terrestres » qui implique «une autonomisation et une réduction du champ du gouvernement des hommes », lequel se trouverait ainsi déchargé des fins ultimes. Les premiers chapitres montrent de quelle façon le cœur du programme théologique de la scolastique produit une description de la nature humaine, dotée d'une dignité propre, en traitant de l'universalité de l'humanité marquée par le péché originel (chap. 1), le 
salut auquel elle est destinée (chap. 2) et le bonheur auquel elle peut aspirer (chap. 3). La dignité, inférieure et néanmoins réelle, reconnue au séjour terrestre réclamerait dès lors un gouvernement limité des hommes, ayant une fonction protectrice, permettant l'accomplissement du salut sans prétendre pouvoir le réaliser. On peut souscrire pleinement à cette analyse, tout en signalant le caractère avant tout négatif de cet effet de structure : la scolastique du XIII ${ }^{\mathrm{e}}$ siècle indique la place possible d'une pensée politique dont elle n'a pas fixé les traits de façon positive, faute d'avoir été confrontée à la question du pouvoir.

La rencontre s'est produite, de façon assez brutale, à l'occasion du conflit entre le roi de France Philippe le Bel et le pape Boniface VIII, chacun des camps en présence mobilisant des théologiens professionnels. Les trois derniers chapitres du livre illustrent la montée d'une « idéologie de la souveraineté », revendiquée par l'un ou l'autre camp. Après un chapitre consacré à la construction de doctrines ecclésiologiques, une forme strictement théologique de la pensée de la souveraineté est examinée, sous la forme du privilège exceptionnel qui fonde la doctrine de l'Immaculée Conception, telle que la défend le carme John Baconthorpe. En contrepoint, le dernier chapitre revient sur le pouvoir séculier, sous l'angle de sa « souveraineté économique », en suivant la justification du droit éminent du prince sur les biens des sujets, que Philippe le Bel pratiqua abondamment. En dépit d'un risque de confusion quant au statut exact de l' « État-république » des scolastiques, l'ouvrage apporte une contribution importante à l'histoire de la pensée politique, en faisant apparaître la fonction limitatrice qu'a remplie, dans la longue durée, la théologie scolastique, à l'égard des tentations de sacraliser le pouvoir et les concepts politiques. C'est une pièce que l'on peut situer dans un ensemble plus vaste, en tenant compte des travaux plus anciens d'Alain Boureau sur la sacralité du pouvoir royal, et des relations complexes qu'il entretient avec l'œuvre d'Ernst Kantorowicz ${ }^{6}$. Se découvre alors une complémentarité entre les deux perspectives. L'histoire d'un État souverain, dont le droit et la liturgie fournissent les expressions privilégiées, bute sur les résistances et limitations imposée par la raison scolastique, dont Kantorowicz n'a guère tenu compte. [213]

Je passerai plus rapidement sur le contenu du troisième volume de la série, De vagues individus, qui pose moins de difficultés de lecture mais dont l'analyse détaillée emmènerait trop loin. Il aborde plus directement la « science de l'homme » scolastique, qui est au centre du projet d'ensemble, sous un angle encore une fois inattendu. Plutôt que de célébrer l'avancée triomphante du sujet, Alain Boureau préfère ici « œuvrer à une histoire médiévale du sentiment de l'aliénation » (DVI, 14), en s'intéressant avant tout aux dualités dans lesquelles est pris l'humain. Le livre se divise en deux parties dont la première n'a rien de kaléidoscopique. L'enquête s'attache de façon continue à la question des puissances de l'âme, c'est-à-dire à l'articulation des facultés mentales à la

${ }^{6}$ Alain Boureau, Le Simple corps du roi. L'impossible sacralité des souverains français, хve-хvIIe siècles, Paris, Les Editions de Paris, 1988 ; Id., Histoires d'un historien. Kantorowicz, Paris, Gallimard, 1990. 
totalité de l'âme humaine. La question de l'association de l'âme au corps - dualité majeure s'il en est -, y est en quelque sorte abordée de biais. Pour cette raison, une mise en place plus consistante des enjeux de l'ouvrage aurait été utile. Suivant tout d'abord les multiples classifications des fonctions mentales au XII ${ }^{\mathrm{e}}$ siècle, deux courants sont présentés. Le premier, partant d'Hugues de Saint-Victor, offre une piste qualifiée de «naturaliste », qui associe à l'essence de l'âme les trois « pulsions » platoniciennes - rationnelle, concupiscible et irascible - qui traversent l'humain sans y être logées de façon stable. Une autre pente, dont le point de départ est ici fixé chez les cisterciens Isaac de l'Étoile et Alcher de Clairvaux, auteur d'un De spiritu et anima qui fut rapidement attribué à saint Augustin, considère prioritairement l'âme et ses forces en regard de la Trinité divine. Sans remettre en question l'existence de ces deux courants, leur stricte assignation me semble trop fortement marquée. Hugues de Saint-Victor est ici sollicité pour son seul Didascalicon, alors que l'on trouve déjà dans son De sacramentis des considérations importantes sur l'âme comme image et ressemblance divine. Mais l'essentiel du propos tient à la thématisation nouvelle produite par les premières générations de théologiens universitaires, qui associent à l'analyse de l'âme humaine la catégorie aristotélicienne de puissance. Après des pages fortes consacrées notamment à Alexandre de Hales, Guillaume d'Auvergne ou Philippe le Chancelier, l'auteur qui est mis au premier plan est le franciscain Jean de La Rochelle. Considéré comme le premier auteur d'une synthèse achevée sur l'âme humaine, son anthropologie, dans le sillage de laquelle s'inscrit tout un courant franciscain, est mise en regard de celle de Thomas d'Aquin. Tandis que le premier considère l'âme comme une substance créée, à la fois autonome et dépendante, elle est pour le second une forme engagée dans un corps ; le dominicain va au bout d'une identification des deux sens distincts d'anima, comme principe d'animation corporelle et comme esprit divinement infusé. Autre opposition, Jean fait jouer un rôle majeur aux "passions de l'âme », tandis que Thomas les cantonne dans une fonction inférieure, accordant une importance supérieure à la notion d'habitus - terme qu'Alain Boureau choisi de traduire par « qualification » (DVI, 169) - chargée d'assurer la continuité du sujet humain.

La seconde partie du livre (qui débute réellement au chapitre 6, le précédent, sur l'habitus, appartenant davantage à l'enquête initiale) multiplie les explorations de la dualité dans laquelle est prise la condition humaine, à travers le thème de l'image divine, de l'ange gardien comme double personnel ou de la sexuation. Ce n'est qu'en fin de course qu'apparaît une question attendue : la transposition du [214] concept de "personne » de la Trinité divine à l'humain singulier dans la seconde moitié du XIII siècle - Olivi étant à nouveau un auteur crucial. Le résultat n'en est pas décrit comme une stabilité, mais au contraire sous l'angle d'une «substitution de l'être humain par luimême ». Si l'on peut regretter l'absence d'une récapitulation d'ensemble, il faut saluer la présence d'un dossier de textes qui permet de suivre plus aisément le cheminement de la première partie de l'ouvrage. 
Vu l'ampleur des trois ouvrages soumis à examen, j'ai laissé en retrait les critiques de détails qui peuvent être faites à certaines des démonstrations. Sans faire reproche à l'auteur de sa façon de procéder, on pourra trouver, dans ses chemins de traverse, certains raccourcis trop rapides, des ascensions un peu abruptes, et au contraire d'autres flâneries un peu longues. La finition aurait pu être mieux soignée ; on passera sur quelques coquilles et des lapsus qui se corrigent facilement. Le choix des textes et des auteurs étudiés est revendiqué. Néanmoins, quelques absents auraient sûrement mérité d'être invités au banquet. Roger Bacon est presque totalement négligé tandis qu'Albert le Grand n'occupe qu'une place mineure au regard de son importance historique. Enfin, la diversité des sujets traités pouvait justifier qu'un index des matières complète l'index des personnes.

Il est plus important de considérer les ambiguïtés de fond présentes dans chacun des volumes, qui contribuent d'ailleurs à leur fécondité. En tête de ses trois livres, l'auteur proteste de son incompétence de philosophe et revendique un statut d'historien. Il est permis de douter que ces déclarations aient la moindre pertinence. Le travail accompli dans la Raison scolastique est d'une nature particulière qu'il faut tenter de qualifier. Mis à part un chapitre résumant les grandes tendances de l'histoire politique médiévale, il s'agit d'une stricte lecture interne de la pensée scolastique. Dans le même temps, l'approche buissonnière s'écarte du canon de l'histoire des doctrines telle qu'elle se pratique dans les facultés de philosophie. Il faut prendre au sérieux les revendications de défense et illustration de la scolastique comme «science de l'homme », dont il s'agirait de réactiver la vitalité, sans aucunement la tirer vers une théologie, et les déclarations de sympathie envers « nos frères scolastiques » (DVI, 16). Leur démarche permettrait, par exemple, ce que la parcellisation des sciences humaines interdit : " une audace ou une ingénuité qui puissent oser parler sans retenue des problèmes violents et insolubles de l'être humain » (DVI, 15). D'autres indices vont dans le même sens, tel le portrait de Jean de La Rochelle en «penseur de la contingence », avantageusement comparé à Heidegger (DVI, 115). De même, il faut noter l'effort fait pour traduire les notions médiévales en équivalents porteurs de sens pour les oreilles contemporaines, quitte à rompre avec des habitudes établies. Ainsi, par exemple, le couple aristotélicen generatio et corruptio n'est pas rendu, comme c'est l'usage, par son simple décalque français, mais par les notions bien plus fortes de «production » et «destruction ». L'ambiguïté de fond de La Religion de l'État révèle qu'Alain Boureau prend au sérieux la pensée politique dont la scolastique était porteuse, comme gouvernement limité et protecteur des individus, de même qu'il y trouve plus généralement des outils pour une pensée globale de l'être humain, une « interrogation radicale » dont seules la littérature et la psychanalyse sont chargées dans la culture contemporaine (DIV, 15). La description exacte [215] de son travail devrait donc amener à parler d'une anthropologie philosophique, qui utilise les moyens de l'histoire des doctrines, avec des scrupules 
d'historien, sensible à la chronologie et au contexte des concepts abordés, mais en vue d'une réflexion neuve et vivante.

Un des fils de l'œuvre d'Alain Boureau, qui ne se traduit pas sous forme de livres mais d'articles souvent brefs, passe depuis des années par un dialogue unique qu'il est parvenu à instaurer avec la psychanalyse. Des chroniques, parues autrefois dans la Nouvelle revue de psychanalyse et désormais dans Penser/rêver, font dialoguer des interrogations analytiques avec des textes scolastiques ${ }^{7}$. C'est à la lumière de cette partie de son œuvre qu'il faut comprendre ses récents ouvrages, comme une tentative de trouver dans la scolastique des moyens renouvelés de poser les « interrogations radicales ». La pensée médiévale n'est pas abordée et évaluée en tant qu'elle serait la source d'une tradition, mais au contraire comme réservoir de pistes oubliées qui apparaissent, une fois remise en lumière, avec l'éclat d'une paradoxale nouveauté. Dans chacun des volumes, la présentation que fait Alain Boureau de la scolastique est marquée par des tensions entre exigences contradictoires et complémentaires. De même, son propre travail est pris entre une exigence d'historien et un désir d'anthropologue philosophe. Cette situation unique explique la richesse, et parfois aussi la difficulté que présentent ses livres.

Sylvain Piron

EHESS

7 - Une quinzaine d'articles de ce type sont parus dans la Nouvelle Revue de Psychanalyse, de 1986 à 1995 , et dans Penser/rêver, depuis 2002. 